\title{
EQUIVALENCE OF EULERIAN AND LAGRANGIAN WEAK SOLUTIONS OF THE COMPRESSIBLE EULER EQUATION WITH SPHERICAL SYMMETRY
}

By Kiyoshi Mizohata

\section{Introduction}

One of the typical equations in fluid mechanics is the compressible Euler equation which describes the inviscid motion of an isentropic gas. The compressible Euler equation with an external force $\vec{f}$ in $R^{n}$ is the $(n+1) \times(n+1)$ system of conservation laws,

$$
\left\{\begin{array}{l}
\rho_{t}+\sum_{j=1}^{n} \frac{\partial}{\partial x_{j}}\left(\rho u_{j}\right)=0 \\
\left(\rho u_{\imath}\right)_{t}+\sum_{j=1}^{n} \frac{\partial}{\partial x_{j}}\left(\rho u_{\imath} u_{j}+\delta_{i j} P\right)=\rho f_{\imath}, \quad(i=1,2, \cdots, n)
\end{array}\right.
$$

where $\rho$ is the density, $\vec{u}={ }^{t}\left(u_{1}, u_{2}, \cdots, u_{n}\right)$ is the velocity, $P$ is the scalar pressure with $\delta_{i}$, the Kronecker delta and $\vec{f}(t, x)={ }^{t}\left(f_{1}, f_{2}, \cdots, f_{n}\right)$ is the external force. For an isentropic gas $P$ satisfies

$$
P=a^{2} \rho^{\gamma},
$$

where $a>0$ and $\gamma \geqq 1$ are given constants.

Let us consider the initial and boundary value problem for (1.1) in $t \geqq 0$, $x \in \Omega \subset R^{n}$ with the following conditions.

$$
\begin{gathered}
\vec{u}(0, x)=\vec{u}_{0}(x), \quad \rho(0, x)=\rho_{0}(x), \\
\vec{u} \cdot \vec{n}=0 \quad \text { if } x \in \partial \Omega,
\end{gathered}
$$

where $\vec{n}$ is the unit vector normal to the boundary.

$\vec{u}(t, x)$ and $\rho(t, x)$ are called weak solutions of (1.1), (1.3) and (1.4) if $u_{\imath}, \rho$ $\in L^{\infty}((0, T) \times \Omega) \quad(i=1,2, \cdots, n), \quad f_{i}(t, x) \in L_{l o c}^{1}((0, T) \times \Omega) \quad(i=1,2, \cdots, n)$ and if they satisfy the following $n+1$ integral identities

Received August 19, 1993. 


$$
\left\{\begin{array}{l}
\int_{0}^{T} \int_{\Omega} \rho \phi_{t}+\sum_{j=1}^{n} \rho u_{j} \phi_{x_{j}} d x d t+\int_{\Omega} \rho_{0}(x) \phi(0, x) d x=0 \\
\int_{0}^{T} \int_{\Omega}\left(\rho u_{\imath}\right) \phi_{i_{t}}+\sum_{j=0}^{n}\left(\rho u_{\imath} u_{j}+\delta_{i j} P\right) \phi_{i_{x}}+\rho f_{i} \phi_{i} d x d t \\
\quad+\int_{\Omega} \rho_{0}(x) u_{0 i}(x) \phi_{i}(0, x) d x=0, \quad(\imath=1,2, \cdots, n)
\end{array}\right.
$$

for any $T>0$ and for any test functions $\phi \in C_{0}^{\infty}([0, T) \times \bar{\Omega})$ and $\phi_{i} \in C_{0}^{\infty}([0, T) \times \Omega)$ $(i=1,2, \cdots, n)$.

Generally speaking, no global weak solution of (1.1) has been known to exist for the case $n \geqq 2$. In [3], we have presented global weak solutions in Lagrangian coordinate with spherical symmetry first for the case $n \geqq 2$. However, it is not obvious that this result also implies the existence of global weak solutions of (1.1). If solutions in Lagrangian coordinate are smooth functions, we can show that $\vec{u}$ and $\rho$ deduced from these solutions satisfy (1.1) by using the chain rule. But if solutions are weak solutions, we must be more careful. In this paper we shall prove that weak solutions in Lagrangian coordinate are weak solutions in Eulerian coordinate at least they are spherically symmetric and that vice versa. Instead of using the chain rule, we use the fact that the Lagrangian transformation is a bi-Lipschitz homeomorphism to prove that $(\vec{u}, \rho)$ is also weak solution of (1.1). This is the main idea of Wagner [4]. He has showed the equivalence for the Cauchy problem in one space dimension. In this paper we shall give the detailed proof of this equivalence for the more general case.

Assumption. $\vec{f}$ is supposed to be spherically symmetric, that is, there exists a scalar function $f$ such that

$$
f_{\imath}=\frac{x_{\imath}}{|x|} f(t,|x|) \quad(i=1,2, \cdots, n) .
$$

We look for the solutions with spherical symmetry, say, of the form

$$
u_{i}(t, x)=\frac{x_{\imath}}{|x|} \tilde{u}(t,|x|) \quad(i=1,2, \cdots, n), \rho(t, x)=\hat{\rho}(t,|x|),
$$

with the initial condition

$$
u_{i}(0, x)=\frac{x_{\imath}}{|x|} \tilde{u}_{0}(|x|) \quad(i=1,2, \cdots, n), \rho(0, x)=\hat{\rho}_{0}(|x|) .
$$

Hereafter, we put $\Omega=\{x ;|x|>A\}$ with $A>0$ since we only deal with the solutions with spherical symmetry. Then, denoting

$$
r=|x|, \quad \tilde{\rho}=\hat{\rho} r^{n-1},
$$

(1.1), (1.3) and (1.4) become 


$$
\left\{\begin{array}{l}
\tilde{\rho}_{t}+(\tilde{\rho} \tilde{u})_{r}=0, \\
(\tilde{\rho} \tilde{u})_{t}+\left(\tilde{\rho} \tilde{u}^{2}+P r^{n-1}\right)_{r}=\frac{n-1}{r} P r^{n-1}+\tilde{\rho} f, \\
\tilde{u}(0, r)=\tilde{u}_{0}(r), \quad \tilde{\rho}(0, r)=\tilde{\rho}_{0}(r), \\
\tilde{u}(t, A)=0 .
\end{array}\right.
$$

We call $\tilde{u}$ and $\tilde{\rho}$ weak solutions of (1.10), (1.11) and (1.12) if they satisfy

$$
\left\{\begin{array}{l}
\int_{0}^{T} \int_{A}^{\infty} \tilde{\rho} \tilde{\phi}_{t}+\tilde{\rho} \tilde{u} \tilde{\phi}_{r} d r d t+\int_{A}^{\infty} \tilde{\rho}_{0}(r) \tilde{\phi}(0, r) d r=0 \text { for any } \tilde{\phi} \in C_{0}^{\infty}([0, T) \times[A, \infty)), \\
\int_{0}^{T} \int_{A}^{\infty} \tilde{\rho} \tilde{u} \tilde{\phi}_{t}+\left(\tilde{\rho} \tilde{u}^{2}+P r^{n-1}\right) \tilde{\psi}_{r}+\left(\frac{n-1}{r} P r^{n-1}+\tilde{\rho} f\right) \tilde{\psi} d r d t \\
\quad+\int_{A}^{\infty} \tilde{\rho}_{0}(r) \tilde{u}_{0}(r) \tilde{\phi}(0, r) d r=0 \text { for any } \tilde{\phi} \in C_{0}^{\infty}([0, T) \times(A, \infty))
\end{array}\right.
$$

Denote $\Gamma$ the Lagrangian transformation defined by

$$
\Gamma:(t, r) \longrightarrow(t, \xi), \quad \xi=\int_{A}^{r} \tilde{\rho}(t, s) d s .
$$

Suppose that $\Gamma$ is a homeomorphism. Put

$$
\overline{\tilde{\rho}}=\tilde{\rho} \circ \Gamma^{-1}, \quad \bar{v}=\frac{1}{\tilde{\tilde{\rho}}}, \quad \bar{u}=\tilde{u} \circ \Gamma^{-1}, \quad \bar{f}=f \circ \Gamma^{-1} .
$$

Then (1.10), (1.11) and (1.12) become,

$$
\left\{\begin{array}{l}
\bar{v}_{t}-\bar{u}_{\xi}=0, \\
\bar{u}_{t}+\left(\frac{a^{2}}{\bar{v}^{\gamma}}\right)_{\xi} \cdot \frac{1}{r^{(n-1)(\gamma-1)}}=\frac{a^{2} \gamma(n-1) \bar{v}^{1-\gamma}}{r^{n} \cdot r^{(n-1)(\gamma-1)}}+\bar{f},
\end{array}\right.
$$

where

$$
\begin{gathered}
r=A+\int_{0}^{\xi} \bar{v}(t, \zeta) d \zeta, \\
\bar{u}(0, \xi)=\bar{u}_{0}(\xi), \quad \bar{v}(0, \xi)=\bar{v}_{0}(\xi), \\
\bar{u}(t, 0)=0 .
\end{gathered}
$$

We call $\bar{u}$ and $\bar{v}$ weak solutions of (1.16), (1.18) and (1.19) if they satisfy

$$
\left\{\begin{array}{l}
\int_{0}^{T} \int_{0}^{\infty} \bar{v} \bar{\phi}_{t}-\bar{u} \bar{\phi}_{\xi} d \xi d t+\int_{0}^{\infty} \bar{v}_{0}(\xi) \bar{\phi}(0, \xi) d \xi=0 \text { for any } \bar{\phi} \in C_{0}^{\infty}([0, T) \times[0, \infty)) . \\
\int_{0}^{T} \int_{0}^{\infty} \bar{u} \bar{\psi}_{t}+\left(\frac{a^{2}}{\bar{v}^{r}}\right)\left(\frac{1}{r^{(n-1)(\gamma-1)}} \bar{\psi}\right)_{\xi}+\left(\frac{a^{2} \gamma(n-1) \bar{v}^{1-\gamma}}{r^{n} \cdot r^{(n-1)(\gamma-1)}}+\bar{f}\right) \bar{\psi} d \xi d t \\
\quad+\int_{0}^{\infty} \bar{u}_{0}(\xi) \bar{\phi}(0, \xi) d \xi=0 \text { for any } \bar{\phi} \in C_{0}^{\infty}([0, T) \times(0, \infty)) .
\end{array}\right.
$$


We now state our main result.

THEOREM 1.1. (Main result) Suppose that $\bar{u}(t, \xi)$ and $\bar{v}(t, \xi)$ are weak solutions of (1.16) satisfying

$$
\begin{aligned}
& \bar{u}, \bar{v} \in L^{\infty}((0, T) \times(0, \infty)), \\
& 0<\delta_{1} \leqq \bar{v}(t, \xi) \leqq M_{1}, \quad|\bar{u}(t, \xi)| \leqq M_{2} \text { a.e. in }(0, T) \times(0, \infty) .
\end{aligned}
$$

Then $\vec{u}(t, x)$ and $\rho(t, x)$ deduced from $\vec{u}(t, \xi)$ and $\vec{v}(t, \xi)$ by using (1.7), (1.9), (1.15) and (1.17) are weak solutions of (1.1) with spherical symmetry.

Conversely, if $\vec{u}(t, x)$ and $\rho(t, x)$ are weak solutions of (1.1) with spherical symmetry satisfying

$$
\begin{aligned}
& u_{\imath}, \rho \in L^{\infty}((0, T) \times \Omega) \quad(i=1,2, \cdots, n), \\
& 0<\frac{\delta_{2}}{|x|^{n-1}} \leqq \rho(t, x) \leqq \frac{M_{3}}{|x|^{n-1}}, \quad|u(t, x)| \leqq M_{4} \text { a.e. in }(0, T) \times \Omega,
\end{aligned}
$$

then $\bar{u}(t, \xi)$ and $\bar{v}(t, \xi)$ deduced from $\vec{u}(t, x)$ and $\rho(t, x)$ by using (1.7), (1.9), (1.14) and (1.15) are also weak solutions of (1.16).

The proof of this Theorem 1.1 consists of two steps. First, we shall prove the "equivalence of weak solutions of (1.1) and (1.10)". Let us define the meaning of "equivalence" precisely. We first show that if $\vec{u}(t, x)$ and $\rho(t, x)$ are weak solutions of (1.1) with spherical symmetry, then $\tilde{u}(t, r)$ and $\tilde{\rho}(t, r)$, defined by (1.7) and (1.9), are weak solutions of (1.10). Next, we shall show that if $\tilde{u}(t, r)$ and $\tilde{\rho}(t, r)$ are weak solutions of $(1.10)$, then $\vec{u}(t, x)$ and $\rho(t, x)$ defined by (1.7) and (1.9) are weak solutions of (1.1). In this case we call that there exists the equivalence of weak solutions of (1.1) and (1.10). We shall prove this equivalence in section 2 . In the second step we shall prove the equivalence of weak solutions of (1.10) and (1.16), and this completes the proof of our main result. The proof of this equivalence will be given in section 3 .

In [2], we have obtained global weak solutions for (1.16) for the case $\gamma=1$, $A=1$ and $f \equiv 0$ first for the case $n \geqq 2$.

Theorem 1.2. ([2], Theorem 4.2) Let $\gamma=1, A=1$ and $f \equiv 0$. Suppose that initial data $\bar{u}_{0}(\xi)$ and $\bar{v}_{0}(\xi)$ are bounded variation and that $\bar{v}_{0}(\xi) \geqq \delta_{0}>0$ with some positive constant $\boldsymbol{\delta}_{0}$. Then Lagrangian equations (1.16) admit a global weak solution which satisfy

$$
\begin{aligned}
& \bar{u}, \bar{v} \in L^{\infty}((0, T) \times(0, \infty)), \\
& 0<\delta_{0}^{\prime} \leqq \bar{v}(t, \xi) \leqq M^{\prime}, \quad|\bar{u}(t, \xi)| \leqq M^{\prime \prime} \text { a.e. } \text {.n }(0, T) \times(0, \infty),
\end{aligned}
$$

for any $T>0$.

Applying Theorem 1.1 to this result, we can say that global weak solutions 
of (1.1) are constructed.

COROLlary 1.3. Suppose that $\vec{f}=\overrightarrow{0}, \gamma=1$ and $\Omega=\{x ;|x|>1\}$. Let $u_{0}(x)$, $\rho_{0}(x)$ be spherically symmetric and satısfy

$$
\left|u_{0}(x)\right| \leqq M_{0}, \quad \frac{\delta_{0}}{|x|^{n-1}} \leqq \rho_{0}(x) \leqq \frac{M_{0}^{\prime}}{|x|^{n-1}} \text { a.e. in } \Omega,
$$

with $\delta_{0}>0$ and $M_{0}, M_{0}^{\prime}<\infty$. Then there exist global weak solutıons of (1.1).

This is the first result of the existence of global solutions of (1.1) for $n \geqq 2$.

Remark. In [3], we have obtained global weak solutions for (1.16) for the case $\gamma=1, A=1, n=3$ and $f=-M / r^{2}$. This corresponds to the model of an isothermal gas around a star with radius 1 and mass $M$. The similar results apparently hold for this case.

\section{The equivalence of weak solutions of (1.1) and (1.10)}

To show the equivalence of weak solutions of (1.1) and (1.10), we must prove the following theorem.

Theorem 2.1. Suppose that $\vec{u}(t, x)={ }^{t}\left(u_{1}(t, x), u_{2}(t, x), \cdots, u_{n}(t, x)\right)$ and $\rho(t, x)$ are weak solutions of (1.1) with spherical symmetry. Then $\tilde{u}(t, r)$ and $\tilde{\rho}(t, r)$ defined by (1.7) and (1.9) are also weak solutions of (1.10). Conversely, if $\tilde{u}(t, r)$ and $\tilde{\rho}(t, r)$ are weak solutions of $(1.10), \vec{u}(t, x)$ and $\rho(t, x)$ defined by (1.7) and (1.9) are also weak solutions of (1.1).

Proof. First we shall show that weak solutions of (1.1) with spherical symmetry are weak solutions of (1.10). Suppose that $\vec{u}(t, x)$ and $\rho(t, x)$ are weak solutions of (1.1) with spherical symmetry. For any test function $\tilde{\phi}(t, r)$ and $\tilde{\phi}(t, r)$, we put $\phi(t, x)=\tilde{\phi}(t,|x|)$ and $\phi_{i}(t, x)=x_{\imath} /|x| \tilde{\psi}(t,|x|)$. Then they are also test functions. Thus the first equation of (1.5) becomes

$$
\int_{0}^{T} \int_{A}^{\infty}\left(\hat{\rho} \tilde{\phi}_{t}+\hat{\rho} \tilde{u} \tilde{\phi}_{r}\right) r^{n-1} d r d t+\int_{A}^{\infty} r^{n-1} \hat{\rho}_{0}(r) \tilde{\phi}(0, r) d r=0 .
$$

If we put $\tilde{\rho}=\hat{\rho} r^{n-1},(2.1)$ is the same equation as the first equation of (1.13). The $i$-th integral identity of the second equations of (1.5) becomes

$$
\begin{aligned}
& \int_{0}^{T} \int_{A}^{\infty} \frac{x_{\imath}^{2}}{|x|^{2}} \hat{\rho} \tilde{u} \tilde{\psi}_{t}+\sum_{\jmath=1}^{n}\left(\hat{\rho} \tilde{u}^{2} \frac{x_{\imath} x_{\jmath}}{|x|^{2}}+\delta_{\imath \jmath} P\right)\left(\frac{\hat{o}}{\partial x_{\jmath}} \frac{x_{\imath}}{|x|} \tilde{\psi}\right) r^{n-1} d r d t \\
& =-\int_{A}^{\infty} r^{n-1} \hat{\rho}_{0}(r) \tilde{u}_{0}(r) \tilde{\phi}(0, r) d r-\int_{0}^{T} \int_{A}^{\infty} \hat{\rho} r^{n-1} \frac{x_{\imath}^{2}}{|x|^{2}} f \tilde{\psi} d r d t .
\end{aligned}
$$

On the other hand, 


$$
\begin{aligned}
& \sum_{i=1}^{n} \sum_{j=1}^{n}\left(\hat{\rho} \tilde{u}^{2} \frac{x_{\imath} x_{\jmath}}{|x|^{2}}\right)\left(\frac{\partial}{\partial x_{j}} \frac{x_{\imath}}{|x|} \tilde{\psi}\right) r^{n-1} \\
= & \sum_{\imath=1}^{n} \sum_{j=1}^{n}\left(\hat{\rho} \tilde{u}^{2} \frac{x_{\imath} x_{\jmath}}{|x|^{2}}\right)\left(\frac{\delta_{i j}|x|-x_{i}\left(x_{j} /|x|\right)}{|x|^{2}} \tilde{\psi}+\frac{x_{\imath} x_{\jmath}}{|x|^{2}} \tilde{\psi}_{r}\right) r^{n-1} \\
= & \sum_{\imath=1}^{n}\left(\hat{\rho} \tilde{u}^{2} \frac{|x|^{2} x_{\imath}^{2}}{|x|^{4}} \tilde{\psi}_{r}\right) r^{n-1} \\
= & \hat{\rho} \tilde{u}^{2} \tilde{\psi}_{r} r^{n-1} \cdot \\
& \sum_{i=1}^{n} \sum_{j=1}^{n} \delta_{i \jmath} P\left(\frac{\delta_{i j}|x|-x_{i}\left(x_{j} /|x|\right)}{|x|^{2}} \tilde{\phi}+\frac{x_{\imath} x_{\jmath}}{|x|^{2}} \tilde{\psi}_{r}\right) r^{n-1} \\
& =\sum_{\imath=1}^{n} P\left(\frac{|x|-\left(x_{\imath}^{2} /|x|\right)}{|x|^{2}} \tilde{\psi}+\frac{x_{\imath}^{2}}{|x|^{2}} \tilde{\psi}_{r}\right) r^{n-1} \\
& =r^{n-1} P \tilde{\psi}_{r}+\frac{n-1}{r} P r^{n-1} \tilde{\psi} .
\end{aligned}
$$

Summing (2.2) from $\imath=1$ to $n$ and using (2.3) and (2.4), we can derive the second equation of (1.13). Thus we can conclude that $\tilde{u}(t, r)$ and $\tilde{\rho}(t, r)$ are weak solutions of (1.10). This completes the first part of Theorem 2.1.

Next we shall prove the second part. Suppose that $\tilde{u}(t, r)$ and $\tilde{\rho}(t, r)$ are weak solutions of (1.10). If we put $\hat{\rho}=\tilde{\rho} / r^{n-1}$, the first identity of (1.13) becomes

$$
\begin{aligned}
& \int_{0}^{T} \int_{A}^{\infty} \hat{\rho} r^{n-1} \tilde{\phi}_{t}+\hat{\rho} r^{n-1} \tilde{u} \tilde{\phi}_{r} d r d t+\int_{A}^{\infty} \hat{\rho}_{0}(r) r^{n-1} \tilde{\phi}(0, r) d r=0 \\
& \text { for any } \tilde{\phi} \in C_{0}^{\infty}([0, T) \times[A, \infty)) .
\end{aligned}
$$

Let $u_{i}(t, x)$ and $\rho(t, x)$ defined by (1.7), and for any test functions $\tilde{\phi}(t, r)$ and $\tilde{\phi}(t, r)$ we put

$$
\phi(t, x)=\tilde{\phi}(t,|x|), \quad \phi_{i}(t, x)=\frac{x_{\imath}}{|x|} \tilde{\phi}(t,|x|) .
$$

Then (2.5) becomes

$$
\int_{0}^{T} \int_{\Omega} \rho \phi_{t}+\sum_{j=1}^{n} \rho u_{j} \phi_{x_{j}} d x d t+\int_{\Omega} \rho_{0}(x) \phi(0, x) d x=0,
$$

for any test function $\phi \in C_{0}^{\infty}([0, T) \times \bar{\Omega})$ with spherical symmetry. Now we must prove that (2.7) also holds for any test function $\phi \in C_{0}^{\infty}([0, T) \times \bar{\Omega})$. To show it, we use the symmetry of $\vec{u}$ and $\rho$. It is enough to show the next Lemma.

LEMMA 2.2. Let $g(t, x) \in L^{\infty}((0, T) \times \Omega)$ be spherically symmetric and satisfies

$$
\int_{0}^{T} \int_{\Omega} g(t, x) \phi(t, x) d x d t=0
$$

for any test function $\phi(t, x) \in C_{0}^{\infty}((0, T) \times \Omega)$ with sphercal symmetry. Then (2.8) 
holds for any test function whach is not necessarily spherically symmetrac.

Proof. Put $g(t, x)=\tilde{g}(t,|x|)$. If $\phi(t, x)=\tilde{\phi}(t,|x|)$ is a test function with spherical symmetry, we get, from (2.8),

$$
\int_{0}^{T} \int_{A}^{\infty} \tilde{g}(t, r) \tilde{\phi}(t, r) r^{n-1} d r d t=0 .
$$

On the other hand, for any test function $\phi(t, x) \in C_{0}^{\infty}((0, T) \times \Omega)$ which is not necessarily spherically symmetric,

$$
\begin{aligned}
& \int_{0}^{T} \int_{\Omega} g(t, x) \phi(t, x) d x d t \\
& =\int_{0}^{T} \int_{A}^{\infty} \int_{S^{n-1}} \tilde{g}(t, r) \phi(t, r \omega) d \omega r^{n-1} d r d t \\
& =\int_{0}^{T} \int_{A}^{\infty} \tilde{g}(t, r) \int_{S^{n-1}} \phi(t, r \omega) d \omega r^{n-1} d r d t \quad \text { (by Fubini's Theorem) } \\
& =\int_{0}^{T} \int_{A}^{\infty} \tilde{g}(t, r) \ddot{\phi}(t, r) r^{n-1} d r d t \quad\left(\ddot{\phi}(t, r)=\int_{S^{n-1}} \phi(t, r \omega) d \omega\right) \\
& =0
\end{aligned}
$$

since $\ddot{\phi}(t, r)$ is a test function. This completes the proof of Lemma 2.2.

Using Lemma 2.2, we can show that (2.7) holds for any test functions. We also can prove that $u_{\imath}$ and $\rho$ satisfy the second part of (1.5) similarly. Thus we obtain Theorem 2.1.

\section{The equivalence of weak solutions of (1.10) and (1.16)}

The following theorem describes that weak solutions of (1.16) are weak solutions of $(1.10)$.

THEOREM 3.1. Suppose that $\bar{u}(t, \xi)$ and $\bar{v}(t, \xi)$ are weak solutions of (1.16) satisfying

$$
\begin{aligned}
& \bar{u}(t, \xi), \bar{v}(t, \xi) \in L^{\infty}((0, T) \times(0, \infty)), \\
& 0<\delta_{1} \leqq \bar{v}(t, \xi) \leqq M_{1}, \quad|\bar{u}(t, \xi)| \leqq M_{2} \text { a.e. in }(0, T) \times(0, \infty) .
\end{aligned}
$$

Then $\tilde{u}(t, r)$ and $\tilde{\rho}(t, r)$ defined by (1.15) and (1.17) are weak solutions of (1.10).

Proof. For the sake of simplicity, we restrict ourselves to the case $\gamma=1$. Suppose that $\bar{u}(t, \xi)$ and $\bar{v}(t, \xi)$ are weak solutions of (1.16). That is, they satisfy 


$$
\left\{\begin{array}{l}
\int_{0}^{T} \int_{0}^{\infty} \bar{v} \bar{\phi}_{t}-\bar{u} \bar{\phi}_{\xi} d \xi d t+\int_{0}^{\infty} \bar{v}_{0}(\xi) \bar{\phi}(0, \xi) d \xi=0, \\
\int_{0}^{T} \int_{0}^{\infty} \bar{u} \bar{\phi}_{t}+\left(\frac{a^{2}}{\bar{v}}\right) \bar{\phi}_{\xi}+\left(\frac{a^{2}(n-1)}{A+\int_{0}^{\xi} \bar{v}(t, \zeta) d \zeta}+\bar{f}\right) \bar{\psi} d \xi d t \\
\quad+\int_{0}^{\infty} \bar{u}_{0}(\xi) \bar{\psi}(0, \xi) d \xi=0 .
\end{array}\right.
$$

They also satisfy

$$
0<\delta_{1} \leqq \bar{v}(t, \xi) \leqq M_{1}, \quad|\bar{u}(t, \xi)| \leqq M_{2} \text { a. e. in }(0, T) \times(0, \infty) .
$$

Put $\overline{\tilde{\rho}}=1 / \bar{v}$. Then from (3.3),

$$
0<\frac{1}{M_{1}} \leqq \overline{\tilde{\rho}}(t, \xi) \leqq \frac{1}{\delta_{1}}|\bar{u}(t, \xi)| \leqq M_{2} \text { a. e. in }(0, T) \times(0, \infty) .
$$

Now (3.2) becomes

$$
\left\{\begin{array}{l}
\int_{0}^{T} \int_{0}^{\infty} \frac{1}{\overline{\tilde{\rho}}} \bar{\phi}_{t}-\bar{u} \bar{\phi}_{\xi} d \xi d t+\int_{0}^{\infty} \frac{1}{\tilde{\tilde{\rho}}_{0}(\xi)} \bar{\phi}(0, \xi) d \xi=0 \\
\int_{0}^{T} \int_{0}^{\infty} \bar{u} \bar{\psi}_{t}+a^{2} \overline{\tilde{\rho}} \bar{\phi}_{\xi} d \xi d t+\int_{0}^{\infty} \bar{u}_{0}(\xi) \bar{\phi}(0, \xi) d \xi \\
=-\int_{0}^{T} \int_{0}^{\infty}\left(\frac{a^{2}(n-1)}{A+\int_{0}^{\xi} \frac{1}{\tilde{\tilde{\rho}}(t, \zeta)} d \zeta}+\bar{f}\right) \bar{\phi} d \xi d t
\end{array}\right.
$$

Put $r=A+\int_{0}^{\xi} \frac{1}{\overline{\tilde{\rho}}(t, \zeta)} d \zeta$, and denote by $\Lambda$ the mapping $(t, \xi) \rightarrow(t, r)$, namely,

$$
\Lambda(t, \xi)=(t, r) .
$$

We shall show that $\Lambda$ is a bi-Lipschitz homeomorphism. Let us calculate the distribution derivatives of $\Lambda$. For $\bar{\phi} \in C_{0}^{\infty}((0, T) \times(0, \infty))$,

$$
\begin{aligned}
& \left\langle r_{\xi}, \bar{\phi}\right\rangle=-\left\langle r, \bar{\phi}_{\xi}\right\rangle \\
& =-\int_{0}^{T} \int_{0}^{\infty}\left(A+\int_{0}^{\xi} \frac{1}{\overline{\tilde{\rho}}(t, s)} d s\right) \phi_{\xi} d \xi d t=-\int_{0}^{T} \int_{0}^{\infty} \int_{0}^{\xi} \frac{1}{\overline{\tilde{\rho}}(t, s)} d s \bar{\phi}_{\xi} d \xi d t \\
& =-\int_{0}^{T} \int_{0}^{\infty} \int_{s}^{\infty} \frac{1}{\overline{\tilde{\rho}}(t, s)} \bar{\phi}_{\xi} d \xi d s d t \quad \text { (by Fubini's theorem) } \\
& =\int_{0}^{T} \int_{0}^{\infty} \frac{1}{\overline{\tilde{\rho}}(t, s)} \bar{\phi}(t, s) d s d t=\left\langle\frac{1}{\overline{\tilde{\rho}}}, \bar{\phi}\right\rangle .
\end{aligned}
$$

Thus we obtain

$$
\frac{\partial r}{\partial \xi}=\frac{1}{\tilde{\tilde{\rho}}}
$$


Next we calculate $r_{t}$.

$$
\begin{aligned}
& \left\langle r_{t}, \bar{\phi}\right\rangle=-\left\langle r, \bar{\phi}_{t}\right\rangle \\
& =-\int_{0}^{T} \int_{0}^{\infty}\left(A+\int_{0}^{\xi} \frac{1}{\tilde{\tilde{\rho}}(t, s)} d s\right) \bar{\phi}_{t} d \xi d t=-\int_{0}^{T} \int_{0}^{\infty} \int_{0}^{\xi} \frac{1}{\tilde{\tilde{\rho}}(t, s)} d s \bar{\phi}_{t} d \xi d t \\
& =-\int_{0}^{T} \int_{0}^{\infty} \frac{1}{\overline{\tilde{\rho}}(t, s)} \int_{s}^{\infty} \bar{\phi}_{t}(t, \xi) d \xi d s d t \\
& =-\int_{0}^{T} \int_{0}^{\infty} \frac{1}{\overline{\tilde{\rho}}(t, s)} \bar{\psi}_{t}(t, s) d s d t \quad\left(\int_{s}^{\infty} \bar{\phi}(t, \xi) d \xi=\bar{\psi}(t, s)\right) \\
& =-\int_{0}^{T} \int_{0}^{\infty} \bar{u} \bar{\psi}_{s} d s d t+\int_{0}^{\infty}-\frac{1}{\overline{\tilde{\rho}}_{0}(s)} \bar{\phi}(0, s) d s \quad(\text { from }(3.5)) \\
& =\int_{0}^{T} \int_{0}^{\infty} \bar{u} \bar{\phi} d s d t=\langle\bar{u}, \bar{\phi}\rangle .
\end{aligned}
$$

Thus we obtain

$$
\frac{\partial r}{\partial t}=\bar{u}
$$

Therefore we have $r \in W^{1, \infty}$ from (3.4). Now we shall show the following Lemma.

Lemma 3.2. Let $\Omega$ be an open convex subset of $R^{n}$. Then

$$
W^{1, \infty}(\Omega) \subset \operatorname{Lip}(\Omega) .
$$

Proof. Suppose $\Omega^{\prime}$ is a compact domain in $\Omega$ and satisfies $\operatorname{dist}\left(\partial \Omega, \partial \Omega^{\prime}\right) \geqq$ $\varepsilon_{0}>0$. Let $u \in W^{1, \infty}(\Omega)$. Put $u_{\varepsilon}=\rho_{\varepsilon} * u\left(0<\varepsilon \leqq \varepsilon_{0}\right)$ where $\rho_{\varepsilon}$ is a mollifier. Then

$$
\left|\nabla u_{\varepsilon}(x)\right|=\left|\nabla \int_{\Omega^{\prime}} \rho_{\varepsilon}(x-y) * u(y) d y\right| \leqq\|\nabla u\|_{L^{\infty}} \quad\left(x \in \Omega^{\prime}\right) .
$$

Moreover,

$$
\begin{aligned}
& \left|u_{\varepsilon}(x)-u_{\varepsilon}(y)\right|=\left|\int_{0}^{1} \frac{d}{d \theta} u_{\varepsilon}(y+\theta(x-y)) d \theta\right| \\
& =\left|\int_{0}^{1} \nabla u_{\varepsilon}(x-y) d \theta\right| \leqq\left|\nabla u _ { \varepsilon } \left\|x-y\left|\leqq\|\nabla u\|_{L^{\infty}}\right| x-y \mid .\right.\right.
\end{aligned}
$$

From (3.9) and (3.10) and by using Ascoli-Arzela's theorem, there exists a subsequence of $\left\{u_{\varepsilon}\right\}$ which converges to $\hat{u}$ uniformly on $\Omega^{\prime}$. So $\hat{u}$ is Lipschitz continuous. On the other hand, $u_{\varepsilon}(x)$ converges to $\hat{u}$ in $L_{1}^{\text {loc }}\left(\Omega^{\prime}\right)$ as $\varepsilon \rightarrow 0$. Then it follows that $u=\hat{u}$ on $\Omega^{\prime}$. Since $\varepsilon_{0}$ is an arbitrary small positive number and the estimates of (3.9) and (3.10) are independent of $\varepsilon_{0}, u$ is Lipschitz continuous in $\Omega$. 
From Lemma 3.2, $r$ is Lipschitz continuous with respect to $\xi$ and $t$. Thus we obtain that $\Lambda$ is Lipschitz continuous. Moreover, an easy calculation shows that $\Lambda$ is a homeomorphism by (3.4). Next we shall show that $\Gamma$ (inverse of 1) is Lipschitz continuous. Due to (3.7) and (3.8), we get

$$
\begin{aligned}
& \left(\begin{array}{c}
t-t^{\prime} \\
r-r^{\prime}
\end{array}\right) \\
& =\left(\int_{0}^{1} \bar{u}\left(\theta(t, \xi)+(1-\theta)\left(t^{\prime}, \xi^{\prime}\right)\right) d \theta \int_{0}^{1} \frac{0}{\overline{\tilde{\rho}}\left(\theta(t, \xi)+(1-\theta)\left(t^{\prime}, \xi^{\prime}\right)\right)} d \theta\right)\left(\begin{array}{c}
t-t^{\prime} \\
\xi-\xi^{\prime}
\end{array}\right) .
\end{aligned}
$$

Using (3.4), we obtain

$$
\left|\xi-\xi^{\prime}\right| \leqq M_{1}\left(\left|r-r^{\prime}\right|+M_{2}\left|t-t^{\prime}\right|\right) .
$$

From (3.12), it follows that $\Gamma$ is also Lipschitz continuous. The following Lemma plays an important role in this section.

LEMMA 3.3. Let $X, Y$ be measurable subsets of $R^{n}$ and $P$ be a mapping from $X$ onto $Y$. If $P$ is Lipschiz continuous, JP (Jacobian) is defined a.e.. Moreover, if $P$ is bi-Lipschitz homeomorphısm and satısfies $|J P| \geqq \delta$ a.e. for some $\delta>0$, then we have, for any $u(x) \in L^{1}\left(R^{n}\right)$,

$$
\int_{X} u(x) d x=\int_{Y} \frac{u \circ P^{-1}}{J P} d y .
$$

For the proof, see [1].

Note that $\bar{u}$ and $\bar{v}$ also satisfy (3.2) for Lipschitz test functions $\bar{\phi}$ and $\bar{\phi}$ instead of smooth test functions. We can prove it by using the mollifier.

Consider an inhomogeneous partial differential equation

$$
\bar{F}_{t}+\bar{G}_{\xi}=\bar{H}
$$

in $(0, T) \times(0, \infty)$ with initial condition $\bar{F}(0, \xi)=\bar{F}_{0}(\xi)$. It's weak form is written as

$$
\int_{0}^{T} \int_{0}^{\infty} \bar{F} \bar{\phi}_{t}+\bar{G} \bar{\phi}_{\xi}+\bar{H} \bar{\phi} d \xi d t+\int_{0}^{\infty} \bar{F}_{0}(\xi) \bar{\phi}(0, \xi) d \xi=0 .
$$

Using Lemma 3.3 and (3.7), (3.15) becomes

$$
\begin{aligned}
& \int_{0}^{T} \int_{A}^{\infty}\left((\bar{F} \circ \Gamma)\left(\bar{\phi}_{t} \circ \Gamma\right)+(\bar{G} \circ \Gamma)\left(\bar{\phi}_{\xi} \circ \Gamma\right)\right)(\overline{\tilde{\rho}} \circ \Gamma) d r d t \\
& \quad+\int_{0}^{T} \int_{A}^{\infty}(\bar{H} \circ \Gamma)(\bar{\phi} \circ \Gamma)(\overline{\tilde{\rho}} \circ \Gamma) d r d t \\
& \quad+\int_{t=0}\left(\bar{F}_{0} \circ \Gamma\right)(\bar{\phi} \circ \Gamma)\left(\overline{\tilde{\rho}}_{0} \circ \Gamma\right) d r=0 .
\end{aligned}
$$

Put $\tilde{\phi}=\bar{\phi} \circ \Gamma, \tilde{F}=\bar{F} \circ \Gamma, \tilde{\rho}=\overline{\tilde{\rho}} \circ \Gamma$, etc. Since $\Gamma$ is a bi-Lipschitz homeomorphism, 
the map $\bar{\phi} \rightarrow \tilde{\phi}=\phi \circ \Gamma$ is a bijection on the set of Lipschitz test functions. This idea is due to D. H. Wagner [3]. We then obtain, using (3.8),

$$
\int_{0}^{T} \int_{A}^{\infty} \tilde{F} \tilde{\rho} \tilde{\phi}_{t}+(\bar{G}+\tilde{\rho} \tilde{u} \tilde{F}) \tilde{\phi}_{r}+\tilde{H} \tilde{\rho} \tilde{\phi} d r d t+\int_{A}^{\infty} \tilde{\rho}_{0}(r) \tilde{F}_{0}(r) \tilde{\phi}(0, r) d r=0 .
$$

Let us substitute the first equation of (3.5). (i.e. $\bar{F}=1 / \overline{\tilde{\rho}}, \bar{G}=-\bar{u}, \bar{H}=0$ ) Then we get a trivial equation. To get a equation for the conservation of mass, we put $\bar{F}=1, \bar{G}=0$ and $\bar{H}=0$. Then we get

$$
\int_{0}^{T} \int_{A}^{\infty} \tilde{\rho} \tilde{\phi}_{t}+\tilde{\rho} \tilde{u} \tilde{\phi}_{r} d r d t+\int_{A}^{\infty} \tilde{\rho}_{0}(r) \tilde{\phi}(0, r) d r=0 .
$$

Similarly if we substitute the second equation of (3.5), using Lipschitz test function $\tilde{\psi}$ instead of $\tilde{\phi}$, (i.e. $\bar{F}=\bar{u}, \bar{G}=a^{2} \tilde{\tilde{\rho}}$ and $\bar{H}=a^{2}(n-1) / r+\bar{f}$ ),

$$
\begin{aligned}
& \int_{0}^{T} \int_{A}^{\infty} \tilde{\rho} \tilde{u} \tilde{\psi}_{t}+\left(\tilde{\rho} \tilde{u}^{2}+a^{2} \tilde{\rho}\right) \tilde{\psi}_{r}+\left(\tilde{\rho} \frac{a^{2}(n-1)}{r}+\tilde{\rho} \tilde{f}\right) \tilde{\psi} d r d t \\
& \quad+\int_{A}^{\infty} \tilde{\rho}_{0}(r) \tilde{u}_{0}(r) \tilde{\psi}(0, r) d r=0 .
\end{aligned}
$$

Recalling that $P=a^{2} \hat{\rho}=a^{2} \tilde{\rho} / r^{n-1}$, we obtain

$$
\begin{aligned}
& \int_{0}^{T} \int_{A}^{\infty} \tilde{\rho} \tilde{u} \tilde{\psi}_{t}+\left(\tilde{\rho} \tilde{u}^{2}+P r^{n-1}\right) \tilde{\psi}_{r}+\left(\frac{(n-1)}{r} P r^{n-1}+\tilde{\rho} \tilde{f}\right) \tilde{\psi} d r d t \\
& \quad+\int_{A}^{\infty} \tilde{\rho}_{0}(r) \tilde{u}_{0}(r) \tilde{\psi}(0, r) d r=0 .
\end{aligned}
$$

Thus we have shown that $\tilde{u}$ and $\tilde{\rho}$ are weak solutions of (1.10).

Next we shall show that the converse is also true.

THEOREM 3.4. Suppose that $\tilde{u}(t, r)$ and $\tilde{\rho}(t, r)$ are weak solutions of (1.10) satisfying

$$
\begin{aligned}
& \tilde{u}, \tilde{\rho} \in L^{\infty}((0, T) \times(A, \infty)), \\
& 0<\delta_{2} \leqq \tilde{\rho}(t, r) \leqq M_{3}, \quad|\tilde{u}(t, r)| \leqq M_{4} \text { a.e. in }(0, T) \times(A, \infty) .
\end{aligned}
$$

Then $\bar{u}(t, \xi)$ and $\bar{v}(t, \xi)$ defined by (1.14) and (1.15) are also weak solutions of (1.16).

Proof. We also restrict ourselves to the case $\gamma=1$ for the sake of simplicity. This proof is similar to above arguments. So we only give the outline of the proof. Suppose that $\tilde{u}(t, r)$ and $\tilde{\rho}(t, r)$ are weak solutions of (1.10). Then we can show

$$
\frac{\partial \xi}{\partial r}=\tilde{\rho}, \quad \frac{\partial \xi}{\partial t}=-\tilde{\rho} \tilde{u} .
$$


The similar computation shows that $\Gamma$ in (1.14) is bi-Lipschitz and homeomorphic and that $\bar{u}(t, \xi)$ and $\bar{v}(t, \xi)$ deduced from $\tilde{u}(t, r)$ and $\tilde{\rho}(t, r)$ by using (1.14) and (1.15) satisfy

$$
\left\{\begin{array}{l}
\int_{0}^{T} \int_{0}^{\infty} \bar{v} \bar{\phi}_{t}-\bar{u} \bar{\phi}_{\xi} d \xi d t+\int_{0}^{\infty} \bar{v}_{0}(\xi) \bar{\phi}(0, \xi) d \xi=0 \\
\int_{0}^{T} \int_{0}^{\infty} \bar{u} \bar{\psi}_{t}+\left(\frac{a^{2}}{\bar{v}}\right) \bar{\psi}_{\xi}+\left(\frac{a^{2}(n-1)}{r}+\bar{f}\right) \bar{\psi} d \xi d t \\
\quad+\int_{0}^{\infty} \bar{u}_{0}(\xi) \bar{\psi}(0, \xi) d \xi=0
\end{array}\right.
$$

LEMMA 3.5.

$$
r=A+\int_{0}^{\xi} \frac{1}{\tilde{\tilde{\rho}}(t, \zeta)} d \zeta
$$

in the Distribution sense.

$$
\begin{aligned}
& \text { Proof. } \\
& \left\langle A+\int_{0}^{\xi} \frac{1}{\overline{\tilde{\rho}}(t, \zeta)} d \zeta, \bar{\phi}(t, \xi)\right\rangle \\
& =A \int_{0}^{T} \int_{0}^{\infty} \bar{\phi}(t, \xi) d t d \xi+\int_{0}^{T} \int_{0}^{\infty} \int_{0}^{\xi} \frac{1}{\tilde{\tilde{\rho}}(t, \zeta)} d \zeta \bar{\phi}(t, \xi) d \xi d t \\
& =A \int_{0}^{T} \int_{0}^{\infty} \bar{\phi}(t, \xi) d t d \xi+\int_{0}^{T} \int_{0}^{\infty} \int_{\zeta}^{\infty} \bar{\phi}(t, \xi) d \xi \frac{1}{\overline{\tilde{\rho}}(t, \zeta)} d \zeta d t \quad \text { (by Fubini's theorem) } \\
& =A \int_{0}^{T} \int_{0}^{\infty} \bar{\phi}(t, \xi) d t d \xi+\int_{0}^{T} \int_{A}^{\infty} \int_{\xi(t, r)}^{\infty} \bar{\phi}(t, \zeta) d \zeta d r d t \\
& =A \int_{0}^{T} \int_{0}^{\infty} \bar{\phi}(t, \xi) d t d \xi+\int_{0}^{T} \int_{0}^{\infty} \int_{A}^{r(t, \zeta)} \bar{\phi}(t, \zeta) d r d \zeta d t \\
& =A \int_{0}^{T} \int_{0}^{\infty} \bar{\phi}(t, \xi) d t d \xi+\int_{0}^{T} \int_{0}^{\infty}(r(t, \zeta)-A) \bar{\phi}(t, \zeta) d \zeta d t \\
& =\langle r(t, \xi), \bar{\phi}(t, \xi)\rangle .
\end{aligned}
$$

This implies (3.24).

From (3.23) and Lemma 3.5, we can conclude that they are also weak solutions of (1.16). Thus we obtain Theorem 3.4.

Combining Theorem 2.1, Theorem 3.1 and Theorem 3.4, we obtain our main result.

Acknowledgements. The author is grateful to Professor S. Ukai for his many helpful comments and encouragements. 


\section{REFERENCES}

[1] H. Federer, Geometric Measure Theory, Springer-Verlag, New York, 1969.

[2] T. Makino, K. Mizohata and S. Ukai, The global weak solutions of the compressible Euler equation with spherical symmetry, Japan J. Indust. Appl. Math., 9 (1992), 431-449.

[3] K. Mizohata, Global weak solutions for the equation of isothermal gas around a star, preprint.

[4] D.H. WAGNER, Equivalence of the Euler and Lagrangian equations of gas dynamics for weak solutions, J. Differential Equations, 68 (1987), 118-136.

Department of INFORMATION SCIENCES

TOKYo Institute OF TECHNOLOGY

OH-OKayama, Meguro-Ku, TOKYo

JAPAN 